\title{
An Optimization Model and Simulation of a Wrecked Plane to Seek for in the Sea
}

\author{
Hong Fang and Xinglong Ren ${ }^{*}$ \\ College of Science, Huazhong Agricultural University, Wuhan, China \\ ${ }^{*}$ Corresponding author
}

\begin{abstract}
It is of great necessity to explore a method to seek for a wrecked plane in the sea. In this paper we think of a static situation and establish a model that can be applied to quickly search the plane fallen into different oceans. Firstly, we develop a parabola model to simulate the process of plane falling. Take the projection of where the plane finally signaled on the sea as the center, and the distance between the two signaling positions plus the horizontal distance of the parabolic movement as radius, we can initially define the most likely falling area. Secondly, we apply Kruskal minimal spanning tree algorithm to minimize the total searching route. Then we regard suspicious neighboring goals as a single zone in different ways and each plane searches only one zone. Considering time cost and fuel cost, a multiobjective programming model is established to optimize the searching scheme. Finally we obtain the minimum cost and time for the whole searching.
\end{abstract}

Keywords-district division; multi-objective programming; Kruskal minimum spanning tree

\section{INTRODUCTION}

In recent years, it is not uncommon that the increasing number of airplane crash issues occurs pretty frequently worldwide. To find the lost plane which fell into the ocean, rescue the survivors in time, and research what cause airline disasters, which contributes to reducing the opportunity of more airplane crash accidents, experts from all over the world have devoted tremendous amount of time and energy in study of searching for disappeared airplanes. Rescue service is a significant action associated with all the public and private resources, aiming at supervising hazards, corresponding with outside, determining the location of victims and transferring them to safer place. The government puts emphasis on rescue service as an indispensable part of emergency rescue system. It guarantees the safety of citizens' lives, and convinces people of their nation's good reputation. Furthermore, it's very critical to save their fellows. Therefore, provided that the government establishes a rescue system with high efficiency and takes appropriate measures promptly, it will make great differences. In this paper, we simulate the process of plane's falling and proposed an efficient approach about how to search for lost planes on the sea.

\section{A PARABOLA MODEL FOR SiMULATING}

Commonly, the plane sends a signal to the ground console every five minutes, including its position, speed, direction etc. We can know the final position of the plane by the last signal it has sent. As Figure I shows, Point $\mathrm{M}$ is where the plane sends the last signal, and Point $\mathrm{N}$ is where it is supposed to send another signal five minutes later.

Regardless of air resistance and other factors, and assume that the plane moves at uniform motion in a straight line between Point $\mathrm{M}$ and Point $\mathrm{N}$. The possible falling point equally distributes on the line $\mathrm{MN}$. If the plane falls from Point $\mathrm{M}$, then it will drop to Point $\mathrm{C}$ in a parabolic track, and if the plane falls from Point N, then it will drop to Point D. Point $\mathrm{C}$ and Point $\mathrm{D}$ are on the sea.

As we do not now the orientation of the plane at last time, it may do parabola movement in any direction[1]. So the possible falling area on the sea is a circle.

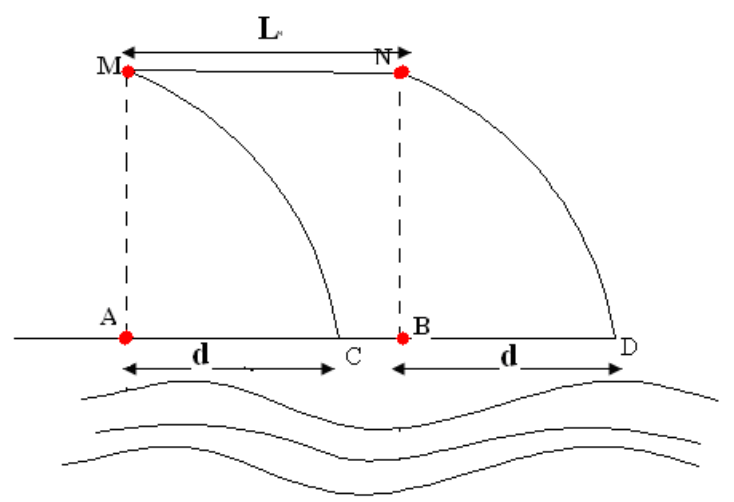

FIGURE I. PARABOLA MODEL

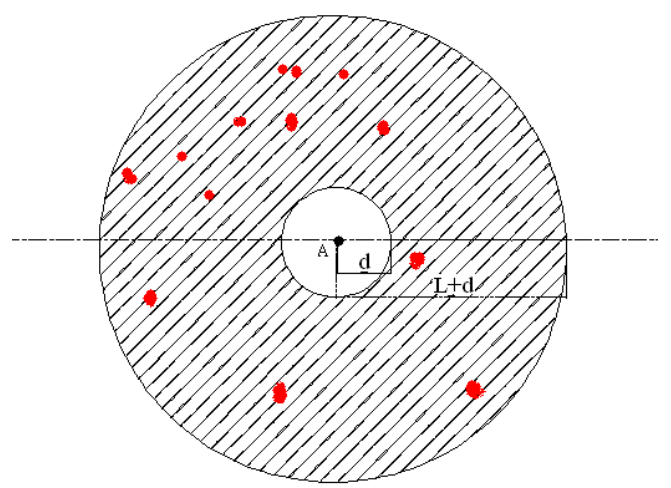

FIGURE II. THE MOST LIKELY FALLING AREA ON THE SEA

Point A and Point B partly represents the projection of Point $\mathrm{M}$ and Point N on the sea. Segment AC and Segment BD 
represent the horizon distance of the parabola movement. Set the length of Segment MN as l, so as Segment $A B$, and the length of Segment AC and Segment BD as d.

Take Point A as center, the length of Segment AC and Segment AD as radius, two circles are drawn, as Figure II shows. The dashed part between the two circles is the possible area where the plane may drop into.

Assume that the flight altitude of the plane $h$ is 10000 meters, and the horizontal flight speed $\mathbf{v}$ is 800 kilometers per hour. According to parabolic equation:

$$
\begin{aligned}
& h=\frac{1}{2} g t^{2} \\
& d=v^{*} t
\end{aligned}
$$

Time needed for falling $t$ is $10 \sqrt{20}$ seconds, and the horizontal distance of the parabolic movement $d$ is $\frac{20000 \sqrt{20}}{9}$ meters.

Assume that the plane flies normally, according to the following equation:

$$
L=v^{*} t_{1}
$$

We can get the horizontal flight distance in five minutes $l$ is $\frac{200000}{3}$ meters.

So the farthest distance the plane can arrive, namely, the radius of the outside circle of the possible falling area $R$ is calculated as the following:

$$
R=L+d
$$

Combining all four equations above, we can obtain the results:

$$
R=\frac{20000 * \sqrt{20}+600000}{9} m \approx 76.604 \mathrm{~km}
$$

According to data available, the detection range of a remote surveillance radar is from 300 kilometers to 500 kilometers, which is much greater than $R$. So if put one that radar at Point A, we can scan all suspicious goals in that area. Red dots in Figure 2 represent all suspicious goals.

\section{KRUSKAL Minimum SPANNING TREE}

Aiming at making the rescue plane rapidly search for all suspicious targets which are detected by radar in the possible region, we adopt the approach which is based on Kruskal minimum spanning tree[2].

Minimum spanning tree is a spanning tree in connected graph with $\mathrm{n}$ nodes. It is the minimal connected sub-graph with all nodes of the original graph, and has the smallest number of edges. To simulate the process of searching for targets in the suspicious region detected by radar, we adopt the dots generated randomly by MATLAB. Those dots simulate the suspect place where plane might fall. Using Kruskal algorithm can guarantee that the searching road is the shortest[3].

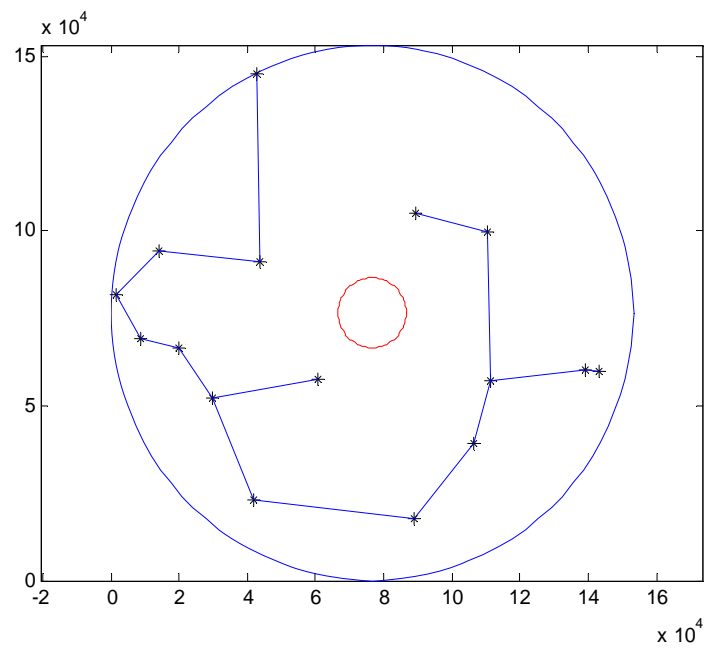

FIGURE III. SUSPICIOUS GOALS AND MINIMAL SPANING TREE

As it is indicated in Figure III, dots where the plane which is simulated randomly might fall present an irregular equal possibility distribution. Take every direction of the flight into consideration, the red dots represent the location when the last time the plane emitted the signal and fell down. The blue circles represent the boundary when the plane have flown for five minutes and arrived at the next signal emitting location. The black dots are generated randomly by MATLAB, representing possible falling locations. The blue segments constitute the minimum spanning tree as the shortest searching road.

\section{Division Of THE SEARCHING AREA}

According to the minimum spanning tree constituted as Figure III shows, we divide near points into different regions. As there are many ways of dividing, we adopt four dividing schemes, the results are showed in Figure IV.

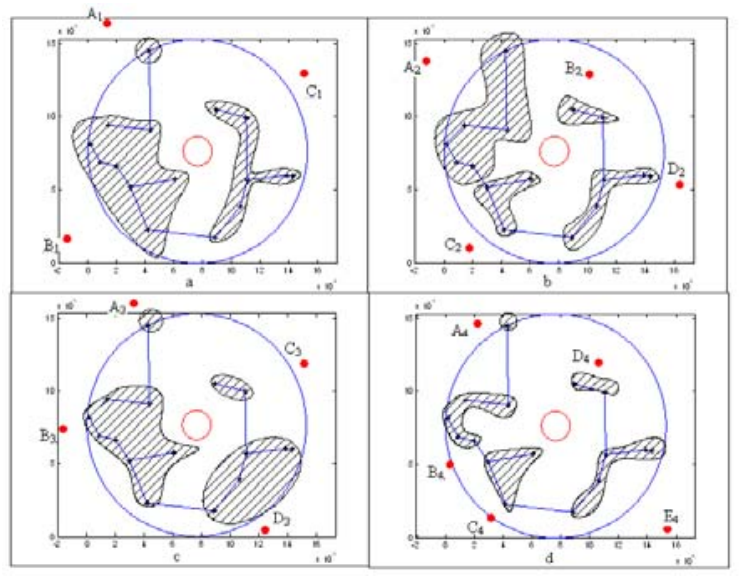

FIGURE IV. SEARCHING AREA DIVIDING SCHEMES 
As we can see in Figure IV, there are respectively three, four, four and five separated regions in every picture. Plus all blue segments, we can obtain the shortest route of searching. Then we can find the goal plane with the fastest speed if only we scan all suspicious targets in shadowed areas[4].

After dividing the regions, assume that airports nearest to every regions can assign rescue planes so as to scan all these regions. Airports are represented by red points in the pictures. As Figure IV shows, there are airports $A_{1}, B_{1}, C_{1}$ in picture a, airports $A_{2}, B_{2}, C_{2}, D_{2}$ in picture $b$, airports $A_{3}, B_{3}, C_{3}, D_{3}$ in picture c, airports $A_{4}, B_{4}, C_{4}, D_{4}, E_{5}$ in picture $d$.

To minimum the cost and make the lasting time for searching as short as possible, we should build a model to compare all these area dividing schemes in order to find out the optimal method. So a multi-objective programming model is established as following.

\section{A MULTI-ObJective Programming Model}

Assume that there are $\mathrm{i}$ kinds of rescue planes and $\mathrm{j}$ areas. $x_{i j}$ means the number of $i_{\text {th }}$ kind of rescue planes assigned to scan the $\mathrm{j}_{\text {th }}$ area. $y_{i j}$ means the scanning length of every $i_{\text {th }}$ kind of rescue plane in $j_{\text {th }}$ area. $z_{i j}$ means the distance between the airport where the $i_{\text {th }}$ kind of rescue planes take off and the farthest point in $\mathrm{j}_{\text {th }}$ area. dist ${ }_{j}$ means the total route needing scanning in the $\mathrm{j}_{\text {th }}$ area. $v_{i}$ means the speed of the $\mathrm{i}_{\text {th }}$ kind of rescue planes. $t_{i}$ means the longest time that the $i_{\text {th }}$ kind of plane can last for flying, $r_{i}$ means the amount of fuel the ith kind of rescue plane consumes in per time-step. $\mathrm{p}$ means the price of per cell fuel. $S_{i}$ means the intrinsic cost of employing the $i_{\text {th }}$ kind of rescue plane, including wages of officers, constructing cost assigned to the specific plane etc.

\section{A. Object Fuction}

To minimum the cost and make the lasting time for searc

Aiming to minimize the time needed[5]. Time needed for each plane is calculated by dividing the distance it flies by its speed. Plus all distances of every single plane, we then get the total time needed. Use the equation below:

$$
\min T=\sum_{i} \sum_{j} \frac{x_{i j} * y_{i j}}{v_{i}}
$$

Aiming to minimize the total cost for searching, which is consisting of fuel cost and intrinsic cost. Fuel cost can be calculated by multiplying the amount of fuel consumed by the price per cell, intrinsic cost is given by collecting data. As a result of lack of data, here we consider the intrinsic cost of every kind of rescue planes is equal, Use the equation below:

$$
\min J=\sum_{i} \sum_{j} \frac{x_{i j} * y_{i j}}{v_{i}} * r_{i} * p+x_{i j} * s_{i}
$$

\section{B. Constraint conditions}

The total distance of all rescue planes assigned to one area can fly should be greater than distance needing scanning of the specific area. This condition is expressed as the equation below:

$$
\sum_{i} x_{i j} * y_{i j} \geq \operatorname{dist}_{j}(j=1,2, \ldots,)
$$

The sum of time for scanning and time for returning should not exceed the plane can last for flying. This condition is expressed as the equation below:

$\sum_{j} \frac{\left(y_{i j}+z_{i j}\right)}{v_{i}} \leq t_{i}(i=1,2, \ldots)$

\section{Data and Results}

By searching the Internet for information, rescue planes we may employ are listed below in Table I:

TABLE I. PARAMETERS OF RESCUE PLANES MAY BE EMPLOYED

\begin{tabular}{llll}
\hline Type & Flight speed & $\begin{array}{l}\text { Maximum flight } \\
\text { range }\end{array}$ & $\begin{array}{l}\text { Fuel consumed } \\
\text { /time-step }\end{array}$ \\
\hline Type 1 & $530 \mathrm{~km} / \mathrm{h}$ & $5500 \mathrm{~km}$ & $4650 \mathrm{~kg} / \mathrm{h}$ \\
Type 2 & $639 \mathrm{~km} / \mathrm{h}$ & $5000 \mathrm{~km}$ & $5100 \mathrm{~kg} / \mathrm{h}$ \\
\hline
\end{tabular}

Because the price of fuel per ton is 4140 yuan at present, using Lingo to solve the model above, we can obtain the result of our model. One is the shortest lasting time: $t_{\min }=1.26 \mathrm{~h}$, and the other is the Minimum rescuing cost: $S_{\min }=27657331$ yuan.

\section{A SUPPLEMENT TO DYNAMIC SIMULATION}

Due to the promoting action of water and wind, a floating object on the sea is always drifting forward. Normally, as for crashed planes fallen into the sea, we simply the issue to the sea surface, and the position of the crashed plane will change irregularly under the impact of oceanic currents and wind pressure. If we can accurately determine the initial location and time of the incident, then its trajectory can be described by modeling.

In order to find out the position of the crashed plane at any time, we need to predict its orientation and speed of drifting.

Assume that the ocean velocity direction relative to the ground is horizontal axis(x), the floater speed relative to the water environment is vertical axis(y). Regard the floater drift velocity as a vector, its decomposition along horizontal axis(x) is $V_{\text {curr }}$, another decomposition along vertical axis(y) is $V_{\text {leeway }}$. Leeway is formulated assuming that the floater on the surface is affected by oceanic currents and wind pressure, making it drift deviated from wind direction. Leeway may turn right clockwise around the wind direction, or turn left anticlockwise wind direction. Set leeway as $\alpha$, then the ocean velocity direction relative to the ground is: 


$$
v_{\text {curr }}=v_{\text {drift }} * \cos \alpha
$$

the floater speed relative to the water environment is:

$$
v_{\text {leeway }}=v_{\text {drift }} * \sin \alpha
$$

Monte-Carlo method is on the basis of statistics, and is well applied in complicated systems. According to equations above, we adopt Monte-Carlo method to simulate the change track of plane.

Use MATLAB to generate initial positions of crashed planes, the result is revealed in Figure V:

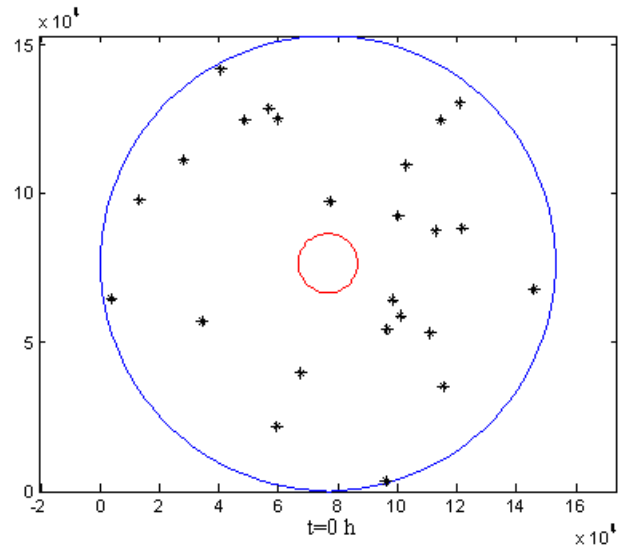

FIGURE V. INITIAL POSITIONS GENERATED BY MATLAB

The figure above simulates the possible positions at original time. As time goes by, the positions of all points turn in all directions. Take 12 hours as a time interval, respectively imitate the positions in 12, 24 and 36 hours based on drift model. The results is shown in Figure VI:
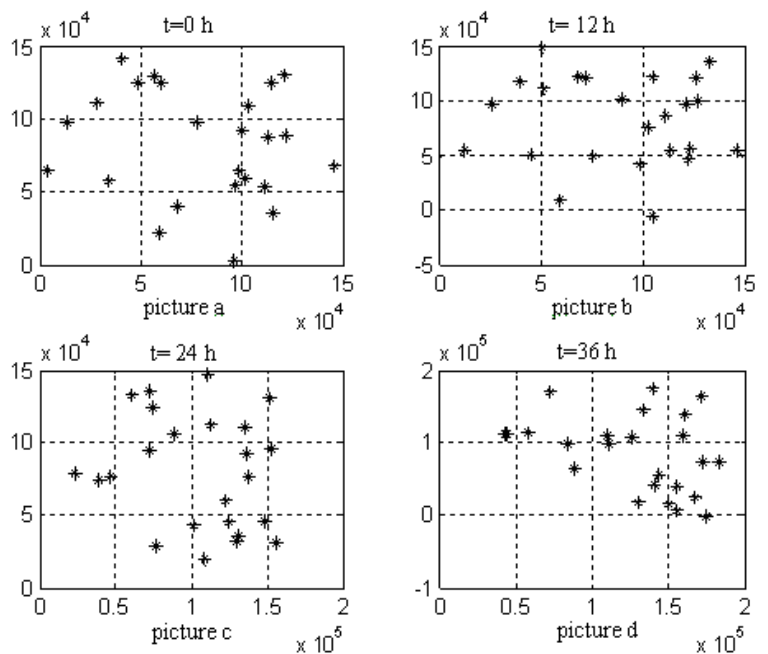

FIGURE VI. POSITIONS IN FURTHER TIMES

We divide the whole zone into square grids with side length of 50000 meters. According to Figure 6, the possibility of the plane's falling into each grid changes as time changes. The possibility is defined as the number of points in each square grid.
Regarding each square grid as a node, we start the searching with nodes with maximum probability. Combining the principle of proximity, if nodes in one probability level searched thoroughly, then the searching plane should find a nearest node with probability in a lower level to restart searching.

Relying on Matlab, we can find out the optimal route for

\begin{tabular}{|c|c|c|c|c|}
\hline$(15000,20000)$ & & (11) & (4). & (12) \\
\hline$(10000,15000)$ & (6) & (10). & (3) & $(\beta)$ \\
\hline$(5000,10000)$ & & & (9) & (2) \\
\hline$(0,5000)$ & & & $(8)$ & (1) \\
\hline$(-5000,0)$ & & & & (13) \\
\hline$(\mathrm{x} . \mathrm{y})$ & $(0,5000)$ & $(5000,10000)$ & $(10000,15000)$ & $(15000,20000)$ \\
\hline
\end{tabular}
searching as is shown in Figure VII.

FIGURE VII. OPTIMAL SEARCHING ROUTE

As calculated, the total route the searching planes need to fly is approximately 1000 kilometers. If needed, several planes should be assigned to search partly.

\section{CONCLUSION}

In this paper, we analyze the method to search for a wrecked plane on the sea in static situation. At first, we seek for a possible area the plane may fall into to limit the searching area, which can reduce searching range and decrease cost in a degree. Secondly, based on Kruskal minimum spanning tree algorithm, we optimize the whole searching route.Of course there are some short comings, such as, In the dropping process of the plane, we ignore air resistance and other factors, but the actual situation may be much more complicated and as few effective data are available in our model establishing model, we just simulate the process on computer, which is not very convincing. And as a supplement, a dynamic simulation is also done, and a feasible search scheme is given.

What's more, we can do some further discussion. Firstly, in parabola model, in order to make sure the possible falling area of the plane more exactly, factors such as air resistance should be involved. Secondly, in district dividing model, more schemes can be listed, which may optimize the searching route for a further step. And at last, we can consider more kinds of planes for searching.

\section{REFERENCES}

[1] Xiao Ming Qi, Ruixuan Wei. Distributed cooperative search methods of multi-UAV based on prediction of moving targets. Systems Engineering and Electronics, 36, pp.2417-2425,2014.

[2] Zhen Liu, Yunan Hu and Jianguo Shi.Modeling of UAV's Dynamic Search and Its Realization Algorithm. Electronics Optics \& Control, 20,pp.38-42,2013. 
[3] Hualin Jiang. Study on Determining Search Area Model for SAR at Sea. Dalian University of Technology, pp.69-75,2011.

[4] Haizheng Liu, Huaici Zhao and Chunyang Zhao. On the Prediction Algorithm of the Search Area for SAR at Sea Based on Monte-Carlo Method.Ship and Ocean Engineering, 39,pp.45-50,2010.

[5] Sung-Pil Hong, Sung-Jin Chol. Optimal search-relocation trade-off in Markovian-target searching. Computers \& Operations Research,36,pp. 2097-2104,2009. 